\title{
Planned Intervention Duration for Study Subject
}

National Cancer Institute

\section{Source}

National Cancer Institute. Planned Intervention Duration for Study Subject. NCI

Thesaurus. Code C70708.

Total estimated length of time during which a clinical study subject is expected to undergo an intervention in the framework of a clinical trial. 